\title{
Novel homozygous mutations in Pakistani families with Charcot-Marie-Tooth disease
}

\author{
Sumaira Kanwall ${ }^{1 \dagger}$, Yu JIn $\mathrm{Choi}^{2+}$, Si On Lim², Hee Ji Choi ${ }^{2}$, Jin Hee Park ${ }^{2}$, Rana Nuzhat ${ }^{3}$, Aneela khan ${ }^{3}$, \\ Shazia Perveen ${ }^{4}$, Byung-Ok Choi ${ }^{*^{*}}$ and Ki Wha Chung ${ }^{2^{*}}$ (D)
}

\begin{abstract}
Background: Charcot-Marie-Tooth disease (CMT) is a group of genetically and clinically heterogeneous peripheral nervous system disorders. Few studies have identified genetic causes of CMT in the Pakistani patients.

Methods: This study was performed to identify pathogenic mutations in five consanguineous Pakistani CMT families negative for PMP22 duplication. Genomic screening was performed by application of whole exome sequencing.

Results: We identified five pathogenic or likely pathogenic homozygous mutations in four genes: c.2599C >T (p.GIn867*) and c.3650G > A (p.Gly1217Asp) in SH3TC2, c.19C > T (p.Arg7*) in HK1, c.247delG (p.Gly83Alafs*44) in REEP1, and c.334G > A (p.Val112Met) in MFN2. These mutations have not been reported in CMT patients. Mutations in SH3TC2, HK1, REEP1, and MFN2 have been reported to be associated with CMT4C, CMT4G, dHMN5B (DSMA5B), and CMT2A, respectively. The genotype-phenotype correlations were confirmed in all the examined families. We also confirmed that both alleles from the homozygous variants originated from a single ancestor using homozygosity mapping.

Conclusions: This study found five novel mutations as the underlying causes of CMT. Pathogenic mutations in SH3TC2, HK1, and REEP1 have been reported rarely in other populations, suggesting ethnic-specific distribution. This study would be useful for the exact molecular diagnosis and treatment of CMT in Pakistani patients.
\end{abstract}

Keywords: Charcot-Marie-Tooth disease (CMT), Consanguinity, Homozygosity, Pakistan, Whole exome sequencing

\section{Background}

Charcot-Marie-Tooth disease (CMT) and related neuropathies are a group of genetically and clinically heterogeneous peripheral neuropathies with a prevalence of approximately 1 in 2500 people [1]. CMT, also called hereditary motor and sensory neuropathy (HMSN), involves the impairment of both sensory and motor nerves, whereas distal hereditary motor neuropathy (dHMN) and hereditary sensory and autonomic

\footnotetext{
*Correspondence: bochoi77@hanmail.net; kwchung@kongju.ac.kr

'Sumaira Kanwal and Yu Jin Choi have contributed equally to this study

${ }^{2}$ Department of Biological Sciences, Kongju National University, 56

Gongjudaehakro, Gongju 32588, Korea

${ }^{5}$ Department of Neurology, Samsung Medical Center, Sungkyunkwan

University School of Medicine, 81 Irwon-ro, Gangnam-gu, Seoul 06351,

Korea

Full list of author information is available at the end of the article
}

neuropathy (HSAN) affect only motor and sensory nerves. Their common clinical phenotypes include progressive distal muscle weakness and atrophy, loss of sensation, and hyporeflexia of the upper and lower limbs [2]. CMT is commonly divided into demyelinating type (CMT1) with a reduced motor nerve conduction velocity (NCV) of less than $38 \mathrm{~m} / \mathrm{s}$, axonal type (CMT2) with normal or slightly reduced NCV of $38 \mathrm{~m} / \mathrm{s}$ or more, and intermediate CMT type (Int-CMT) with $\mathrm{NCV}$ values spanning both CMT1 and CMT2 in a single family $[3,4]$. CMT is often viewed as a monogenic Mendelian disease; however, mutations in more than 130 genes are associated with the development of peripheral neuropathies in an autosomal or X-linked dominant or recessive manner.

Several studies have attempted to determine the underlying causes of CMT [5]. In particular, the use of next

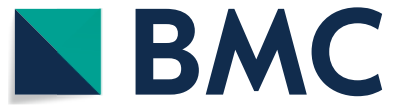

(c) The Author(s) 2021. Open Access This article is licensed under a Creative Commons Attribution 4.0 International License, which permits use, sharing, adaptation, distribution and reproduction in any medium or format, as long as you give appropriate credit to the original author(s) and the source, provide a link to the Creative Commons licence, and indicate if changes were made. The images or other third party material in this article are included in the article's Creative Commons licence, unless indicated otherwise in a credit line to the material. If material is not included in the article's Creative Commons licence and your intended use is not permitted by statutory regulation or exceeds the permitted use, you will need to obtain permission directly from the copyright holder. To view a copy of this licence, visit http://creativecommons.org/licenses/by/4.0/. The Creative Commons Public Domain Dedication waiver (http://creativeco mmons.org/publicdomain/zero/1.0/) applies to the data made available in this article, unless otherwise stated in a credit line to the data. 
generation sequencing (such as whole exome or targeted sequencing) has enhanced the identification of genetic pathogenicities. However, only a few studies have been performed to determine the genetic causes of CMT and related peripheral neuropathies in Pakistan [6-9]. Pakistani patients for whom genetic causes were identified exhibited unusually high frequencies of recessive homozygous mutations. Pedurupillay et al. reported three patients with CMT2S or spinal muscular atrophy with respiratory distress type 1 (SMARD1) with IGHMBP2 mutations [7]. Two of them presented homozygous mutations. Wright et al. reported a homozygous FIG4 variant in four independent patients manifesting combined phenotypes of CMT4J and Yunis-Varón syndrome [9]. Houlden et al. reported several patients with $H S P B 1$ mutations which contained a homozygous mutation in addition to heterozygous mutations [6]. Zambon et al. reported a patient with CMT4B1 with homozygous MTMR2 mutation [8]. The high rates of homozygous mutations in Pakistani patients can be attributed to the relatively frequent occurrence of consanguineous marriages.

This study aimed to determine the genetic causes of CMT or related neuropathies using in Pakistani patients using whole exome sequencing (WES) and subsequent filtering of called variants. We identified five pathogenic or likely pathogenic homozygous mutations in CMTrelated genes. Additionally, we determined that all the observed homozygous mutations originated from a single founder through homozygosity mapping.

\section{Methods}

\section{Subjects}

This study examined five CMT patients and 15 of their unaffected familial members originating from consanguineous Pakistani pedigrees who were negative for the duplication or deletion of the $17 \mathrm{p} 12$ chromosomal region harboring the PMP22 gene (Fig. 1). All participants were recruited from The Children Hospital and Institute of Child Health, Multan, Pakistan, and provided written informed consent. For the minors involved in the study, written consent was provided by their parents. This study was approved by the Institutional Review Boards for Kongju National University (KNU_ IRB_2018-06), Sungkyunkwan University, Samsung Medical Center (2014-08-057-002), COMSATS University Islamabad, Sahiwal (CUI-SWL-09-062-2018), and The Children Hospital and Institute of Child Health, Multan (CHMP-2018-023-0014).

\section{Clinical and electrophysiological assessments}

Motor and sensory impairments, deep tendon reflexes, and muscle atrophy were measured as the clinical information. Onset age was determined by interviewing the patients about when the symptoms, such as distal muscle weakness, foot deformity, or sensory change first appeared. Disease severity was determined using the functional disability scale (FDS) [10]. Motor and sensory conduction velocities of the median, ulnar, peroneal, tibial, and sural nerves were determined by surface stimulation followed by recording using electrodes. Motor nerve conduction velocities (MNCVs) of the median and ulnar nerves were determined by stimulating at the elbow and wrist while recording compound muscle action potentials (CMAPs) over the abductor pollicis brevis and adductor digiti quinti, respectively. In the same way, the $\mathrm{NCVs}$ of peroneal and tibial nerves were determined by stimulation at the knee and ankle while recording CMAPs over the extensor digitorum brevis and adductor

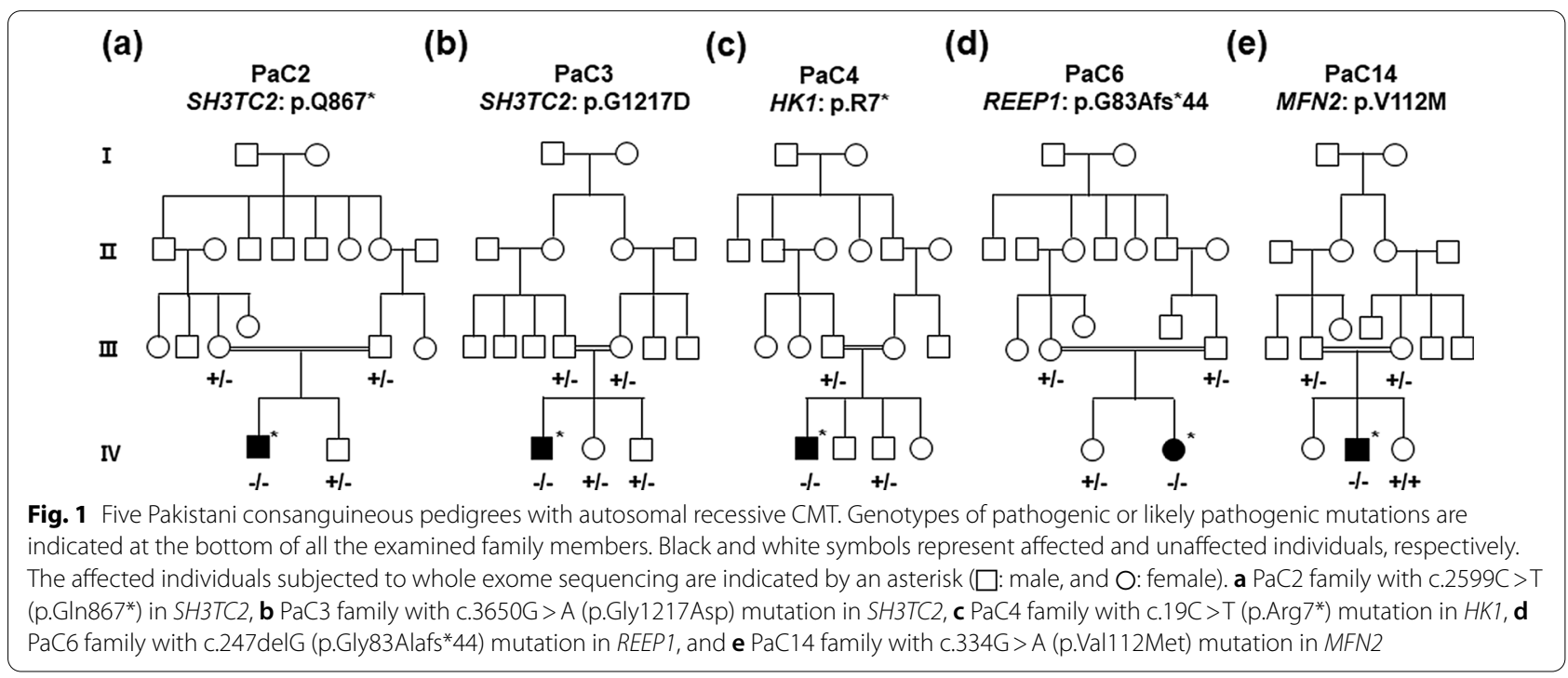


hallucis, respectively. Sensory nerve conduction velocities (SNCVs) and sensory nerve action potential (SNAP) amplitudes were obtained over a finger-wrist segment from the median and ulnar nerves by the orthodromic method and were also recorded for sural nerves. Electromyography was carried out with a concentric needle electrode. Magnetic resonance imaging (MRI) of the brain and spinal cord were carried out using a 1.5-T system (Siemens Healthineers, Erlangen, Germany).

\section{DNA purification and pedigree analysis}

Genomic DNA was purified from whole blood using the HiGene Genomic DNA Prep Kit (Biofact, Daejeon, Korea). Paternity was confirmed for all the examined families by PCR amplification of STR markers using the PowerPlex Fusion System (Promega, WisconsinMadison, USA) followed by the resolution of the PCR products on a SeqStudio genetic analyzer (Life Technologies-Thermo Fisher Scientific, Foster City, CA, USA).

\section{Exome sequencing and filtering}

WES was performed for patients in the five examined families. Exome was captured using the SureSelect Human All Exon $50 \mathrm{M}$ kit (Agilent Technologies, Santa Clara, CA, USA), and sequencing was performed using the HiSeq 2000 Genome Analyzer (Illumina, San Diego, CA, USA). The UCSC assembly hg19 (GRCh37) was used as the reference sequence (http://genome.ucsc.edu). Small nucleotide variants (SNVs) were called using the GATK (https://software.broad institute.org/gatk/) and SAMtools (http://samtools.sourceforge.net/). Rare alleles with minor allele frequencies (MAFs) of $<0.01$ were obtained from the 1000 Genomes Project (1000G, http:// www.1000genomes.org/), and the Genome Aggregation Database (gnomAD. https://gnomad.broadinstitute. org/). Candidate variants for the genetic causes were also checked in the dbSNP (http://www.ncbi.nlm.nih.gov/ snp) and ClinVar (https://www.ncbi.nlm.nih.gov/clinv ar/). Pathogenicity of the variants was categorized into five grades (pathogenic, likely pathogenic, uncertain significance, likely benign, and benign) based on the guidelines of the American College of Medical Genetics and Genomics (ACMG) [11]. Pathogenic candidate variants were confirmed by Sanger sequencing using the SeqStudio genetic analyzers (Life Technologies-Thermo Fisher Scientific).

\section{In silico prediction and conservation analysis}

In silico analyses to predict the mutation effect were performed using the programs of MUpro (http://www.ics. uci.edu/ baldig/mutation) [12], PolyPhen-2 (http://genet ics.bwh.harvard.edu/pph2/) [13], PROVEAN (http:// provean.jcvi.org/) [14], and Fahmm (http://fathmm.bioco mpute.org.uk/) [15]. Conservation analysis of the mutation sites was performed using the MEGA-X software, ver. 5.05 (http://www.megasoftware.net/). Genomic evolutionary rate profiling score (GERP) was determined using the GERP + + program (http://mendel.stanford. edu/SidowLab/downloads/gerp/) [16].

\section{Homozygosity mapping}

For the putative pathogenic homozygous variants, homozygosity mapping was performed to determine whether two alleles originated from a single founder. Homozygosity mapping was achieved through haplotyping of SNPs distributed around the corresponding mutations from the WES data of the affected persons using the method outlined by Park et al. [17].

\section{Results}

\section{Clinical manifestations}

In this study, we examined five consanguineous families with CMT. CMT types and clinical phenotypes are listed in Table 1.

In the $\mathrm{PaC} 2$ family, a 6-year-old boy was born with a full term pregnancy from healthy parents. He showed delayed development and frequent falls during walking since he was 3 years old. He did not complain of sensory symptoms; however, his vibration sensation was reduced. Deep tendon reflex at the knee was absent. Scoliosis and feet deformities were observed. No family history of such complaints was recorded. Motor nerve conduction studies revealed prolonged distal latencies and low distal CMAP amplitudes with no reproducible f-wave latencies and wave forms. He did not manifest SNAP responses in the upper and lower extremities. These findings were compatible with demyelinating CMT neuropathy.

In the PaC3 family, a 10-year-old boy born with full term from healthy normal parents had demyelinating CMT. At the age of 3, he was noticed to fall frequently and had difficulty standing from a sitting position. In addition to the CMT phenotype, he manifested scoliosis and short stature. Although he had difficulty in walking, he could walk unaided. Motor nerve conduction studies of median and peroneal nerves showed prolonged distal latencies and low distal CMAP amplitudes of median and peroneal nerves were absent, and those of ulnar and tibial nerves were decreased. Motor and sensory NCVs were decreased at the upper and lower extremities. Brain MRI showed no area of abnormal signal intensity.

In the PaC4 family, an 11-year-old boy born with full-term pregnancy from healthy parents. At the age of 15 months, he was unable to walk without support. His parents first noticed gait disturbance at the age of 2.5 years. No family history of such complaints was recorded. Neurological examination revealed decreased 
Table 1 Clinical characterization of five Pakistani CMT patients

\begin{tabular}{|c|c|c|c|c|c|}
\hline Item/patient (sex) & PaC2:IV-1 (male) & PaC3:IV-1 (male) & PaC4:IV-1 (male) & PaC6:IV-2 (female) & PaC14:IV-2 (male) \\
\hline Gene: mutation & SH3TC2: p.Q867* & SH3TC2: p.G1217D & $H K 1: p . R 7^{*}$ & REEP1: p.G83Afs*44 & MFN2: p.V112M \\
\hline Type & CMT4C & CMT4C & CMT4G & dHMN5B/SMARD1 & CMT2A2B \\
\hline AOE/AOO (year) & $6 / 3$ & $10 / 3$ & $11 / 1$ & $2.5 /<1$ & $7 / 5$ \\
\hline Muscle atrophy & Yes & Yes & Yes & Yes & Yes \\
\hline FDS & 3 & 3 & 3 & 3 & 3 \\
\hline Sensory loss & Yes & Yes & Yes & No & Yes \\
\hline DTR, ankle & Absent & Absent & Absent & Decreased & Absent \\
\hline Foot deformities & Yes & Yes & Yes & Yes & Yes \\
\hline Brain/Spine MRI & ND & Normal brain & Normal spine & Normal brain & ND \\
\hline Other symptoms & Scoliosis & Scoliosis, short stature & - & Mild respiratory distress & Vocal cord hoarseness \\
\hline \multicolumn{6}{|c|}{ Motor nerve conduction studies } \\
\hline Median CMAP (mV) & 1.3 & Absent & ND & Absent & ND \\
\hline Median MNCV (m/s) & 14.1 & Absent & ND & Absent & ND \\
\hline Ulnar CMAP (mV) & 0.8 & 4.1 & ND & Absent & ND \\
\hline Ulnar MNCV (m/s) & 12.8 & 25.0 & ND & Absent & ND \\
\hline Peroneal CMAP (mV) & 0.8 & Absent & ND & Absent & ND \\
\hline Peroneal MNCV $(\mathrm{m} / \mathrm{s})$ & 14.9 & Absent & ND & Absent & ND \\
\hline \multicolumn{6}{|c|}{ Sensory nerve conduction studies } \\
\hline Median SNAP $(\mu \mathrm{V})$ & Absent & 10.6 & ND & 22.0 & ND \\
\hline Median SNCV (m/s) & Absent & 26.0 & ND & 51.3 & ND \\
\hline Ulnar SNAP $(\mu \mathrm{V})$ & Absent & ND & ND & 22.2 & ND \\
\hline Ulnar SNCV (m/s) & Absent & ND & ND & 39.5 & ND \\
\hline Sural SNAP $(\mu V)$ & Absent & 8.8 & ND & 16.3 & ND \\
\hline Sural SNCV (m/s) & Absent & 32.0 & ND & 39.8 & ND \\
\hline
\end{tabular}

AOE: age of examination, AOO: age of onset, CMAP: compound muscle action potential, DTR: deep tendon reflexes, FDS: functional disability scale, MNCV: motor nerve conduction velocity, ND: not done, SNAP: sensory nerve action potential, SNCV: sensory nerve conduction velocity

*indicates putative translational termination

vibration and pain sensation, which was consistent with the results of a sensory nerve conduction study. Deep tendon reflex at the knee was absent, and feet deformities were observed. Lumbo-sacral spine MRI did not reveal any noticeable abnormal signals.

In the PaC6 family, a 2.5-year-old girl born full term from unaffected parents had congenital motor neuropathy. She exhibited delayed development. The affected girl manifested foot deformity and contractures of the distal phalanges before the age of 6 months, and her parents noticed neuromuscular defects before she was 1 year old. She had frequent falls during walking, and mild respiratory distress. Deep tendon reflex at the knee was decreased, and feet deformities were observed. No family history of such complaints was recorded. CMAPs of the median, ulnar, peroneal and tibial nerves were not evoked, but, normal SNAPs and SNCVs were observed in the sensory median, ulnar and sural nerves.

In the PaC14 family, a 7-year-old boy manifested axonal CMT with an onset of 5 years. He had vocal cord hoarseness as an additional symptom. He manifested delayed development. At the age of 5 years, he manifested gait disturbances and had frequent falls during walking. He did not complain of sensory symptom; however, his vibration and position sensation were reduced. Deep tendon reflex at the knee was absent, and feet deformities were observed. No family history of such complaints was recorded.

Identification of novel homozygous pathogenic mutations We identified five pathogenic or likely pathogenic homozygous mutations in the SH3TC2, HK1, REEP1, and MFN2 genes in the examined families by sequencing analysis and rare allele filtering processes (Table 2). All the candidate pathogenic mutations were confirmed by Sanger sequencing (Fig. 2a).

Mutations in the SH3TC2 (MIM 608206) are implicated to the development of recessive CMT4C (MIM 601596) [18] and the relatively mild dominant mononeuropathy of the median nerve (MNMN, MIM 613353) [19]. We identified one novel and one rare homozygous variants of $S H 3 T C 2$ in two families. As the first 


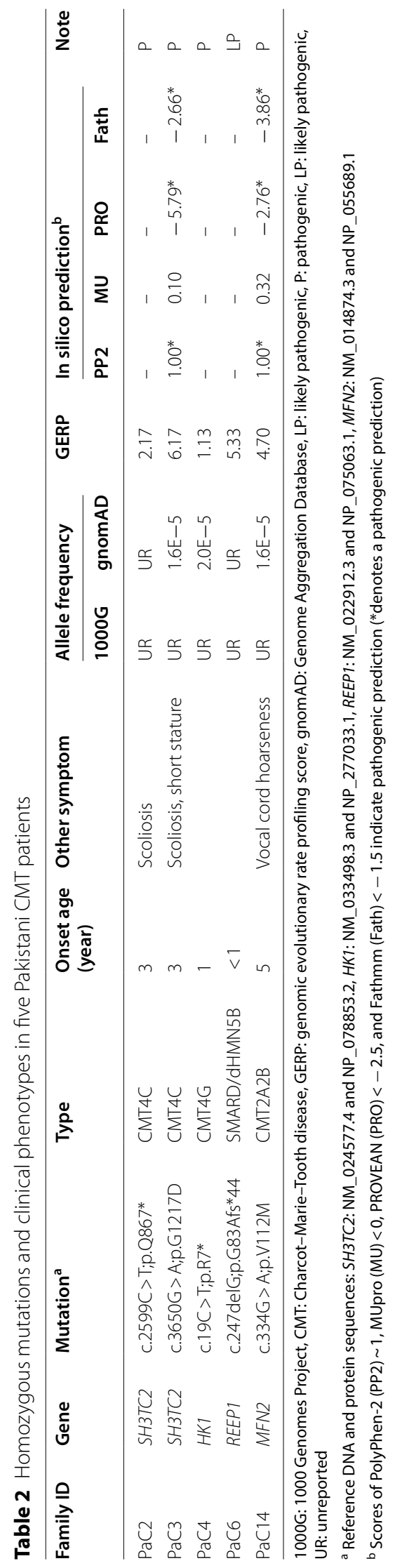




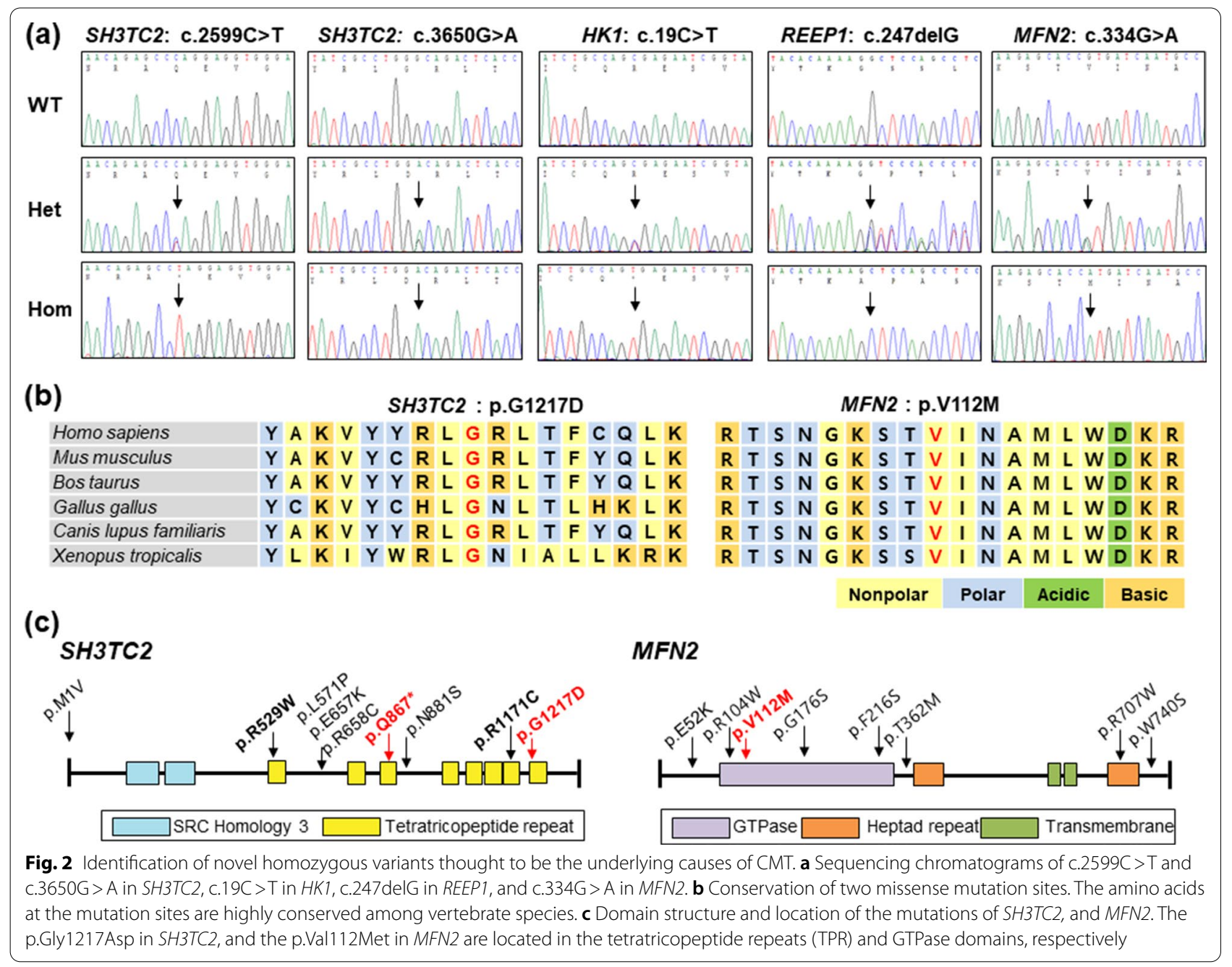

mutation, a novel homozygous c.2599C $>\mathrm{T}$ mutation which results in a stop-gain mutation (p.Gln867*) was identified in a 6-year-old boy (family ID: PaC2). The SH3TC2 mutation was heterozygous in the unaffected parents and a younger brother (Fig. 1a). This mutation has not been reported as pathogenic, nor has it been registered in the public databases of dbSNP, 1000G, or gnomAD. The p.Gln $867 *$ mutation is expected to result in the production of a truncated protein which lacks many tetratricopeptide repeat (TPR) domains. Although p.Gln867* had not been reported yet, several stop-gain mutations, such as p.Gln892*, p.Arg904*, and p.Tyr $943^{*}$, have been reported as the underlying causes of CMT4C $[18,20,21]$. As the second SH3TC2 mutation, c.3650G $>$ A resulting in p.Gly1217Asp was identified in a 10-years-old boy (family ID: PaC3). The unaffected parents and younger sister and brother were heterozygous for the mutation (Fig. 1b). The homozygous p.Gly1217Asp mutation is still not reported as pathogenic, although the same heterozygous variant was recently registered as "uncertain significance" in the ClinVar database. It was registered in the dbSNP (rs758669363) and gnomAD with a very low allele frequency (1.6E-5). The p.Gly1217Asp mutation was located in the highly conserved TPR domain, which plays a putative role in protein-protein interactions (Fig. 2b,c), and in silico prediction analysis using PolyPhen-2, Fathmm, and PROVEAN determined it as being pathogenic.

Only a few cases with homozygous mutations in $H K 1$ have been reported to be associated with autosomal recessive CMT4G (MIM 605285), also called HMSN Russe type $[22,23]$. An 11-year-old boy with demyelinating CMT (family ID: PaC4) harbored a stop-gain mutation. i.e., c.19C $>\mathrm{T}$ (p.Arg7*) in HK1 (MIM 142600), which putatively resulted in a very short premature peptide. The affected boy was homozygous for the mutation and the unaffected father and a younger brother were heterozygous for the mutation (Fig. 1c). This HK1 mutation was not reported as pathogenic in CMT patients. 
The mutation was registered in the dbSNP (rs779250530) and the gnomAD with a very low allele frequency $(2.0 \mathrm{E}-$ 5 ), but all reported individuals were heterozygous for the mutation.

A small number of mutations in REEP1 (MIM 609139) have been reported to cause several neuromuscular disorders, such as the dominant dHMN5B (MIM 614751), also known as distal spinal muscular atrophy type $5 \mathrm{~B}$ (dSMA5B) [24], and dominant spastic paraplegia-31 (SPG31, MIM 610250) [25]. A homozygous splicing mutation was also recently reported in a patient manifesting similar symptoms of spinal muscular atrophy with respiratory distress (SMARD), which has a phenotype similar to that of SMA but with an additional symptom of diaphragmatic palsy [26]. In this study, we identified a homozygous frameshift REEP1 mutation of c.247delG in a 2.5-year-old girl with dHMN (family ID: PaC6). This deletion was expected to produce a truncated premature peptide (p.Gly83Alafs*44). The unaffected parents and an elder sister harbored a heterozygous version of this mutation (Fig. 1d). It has not been registered in any databases, nor has it been reported as a pathogenic mutation.

Most mutations in MFN2 (MIM 608507) are associated with autosomal dominant CMT2A2A (MIM 609260) [27] and CMT6A (MIM 601152) [28], whereas, recessive MFN2 mutations have been rarely reported with more severe and earlier onset CMT2A2B (MIM 617087) [29]. The affected 7-year-old boy in the $\mathrm{PaC} 14$ family harbored a homozygous c.334G > A (p.Val112Met) in MFN2. The unaffected parents and a younger sister harbored a heterozygous version of this mutation (Fig. 1e). The mutation was reported in the gnomAD with a very low frequency (1.6E-5), and was registered as likely pathogenic in the ClinVar database. It was predicted to be pathogenic by the in silico analysis using PolyPhen-2, Fathmm, and PROVEAN, and this mutation was found to be located in the highly conserved GTPase domain among vertebrate species (Fig. 2b,c).

Filtering WES data for the affected individuals of five families revealed several rare functionally significant variants $(\mathrm{MAF}<0.01)$ in CMT-related genes, in addition to the above mentioned five pathogenic or likely pathogenic mutations (Table 3). A homozygous DST c.1933A > G (p.Ile645Val) variant was observed in the $\mathrm{PaC} 4$ patient. The DST mutation was cosegregated with the affected individual. However, in silico analyses using PolyPhen-2 and PROVEAN programs predicted it to be nonpathogenic. DST mutations have been reported to be implicated in HSAN6 (MIM 614653) [30]. Thus, we classified this homozygous variant as a 'variant of uncertain significance (VUS)". All other rare variants were considered as nonpathogenic because they were either nonsegregated with the affected individuals when all the family members were tested by Sanger sequencing or did not fit the inheritance modes of the corresponding genes.

\section{Homozygosity mapping}

We observed homozygous blocks (HBs) at the chromosomal regions including pathogenic or likely pathogenic mutations in all the five affected individuals by the SNP haplotype analysis using WES data (Fig. 3). The lengths of the HBs were approximately $16 \mathrm{Mbp}$ from FGF1 (rs34003) to THG1L (rs2270812) in the PaC2 family with SH3TC2 mutation, $12 \mathrm{Mbp}$ from PKD2L2 (rs700605) to SLC6A7 (rs12653451) in the PaC3 family with SH3TC2 mutation, $38 \mathrm{Mbp}$ from PPYR1 (rs1936339) to NRG3 (rs478010) in the PaC4 family with HK1 mutation, $53 \mathrm{Mbp}$ from CTNNA2 (rs2228460) to MZT2A (rs10182785) in the PaC6 family with REEP1 mutation, and $14 \mathrm{Mbp}$ from $N A D K$ (rs7407) to $C L C N K B$ (rs7517792) in the PaC14 family with MFN2 mutation. Considering the consanguinity in the families, these HBs suggest that both homozygous alleles in each family originated from a single ancestor.

\section{Discussion}

In this study, we performed genetic screening in consanguineous Pakistani CMT families by WES and identified five homozygous mutations in four genes, SH3TC2, HK1, $R E E P 1$, and $M F N 2$, as the underlying cause of recessive CMT. The mutations identified in this study have not been reported in CMT patient, thus far.

SH3TC2, which encodes an SH3 domain and tetratricopeptide repeats-containing protein 2 , is expressed in Schwann cells of peripheral nerves, suggesting a possible role in myelination [31]. Mutations in SH3TC2 cause recessive CMT4C usually concurrent with scoliosis, with the onset ranging from infancy to early teens [18]; however, cases with late onset ( $\leq 30$ years) were also reported [32].

Mutations in $H K 1$ cause recessive CMT4G (HMSNR), mostly found in the Spanish Gypsy patients [22]. Hexokinase 1 encoded by $H K 1$ catalyzes the phosphorylation of glucose. HK1 localizes at the outer membrane of mitochondria (OMM) through a porin-binding domain, and it was suggested that non-OMM-binding HK1 proteins involve in the pathogenesis of CMT4G pathogenesis [22]. Several $H K 1$ mutations are also associated with autosomal dominant retinitis pigmentosa-79 (RP79, MIM 617460), which exhibits variable phenotype with ages of onset ranging from childhood to 70 years [33]. The affected 11-year-old boy with the HK1 mutation did not manifest symptoms of retinitis pigmentosa until his age of examination.

REEP1 encodes a receptor accessory protein 1, suggesting its role in facilitating endoplasmic reticulum 


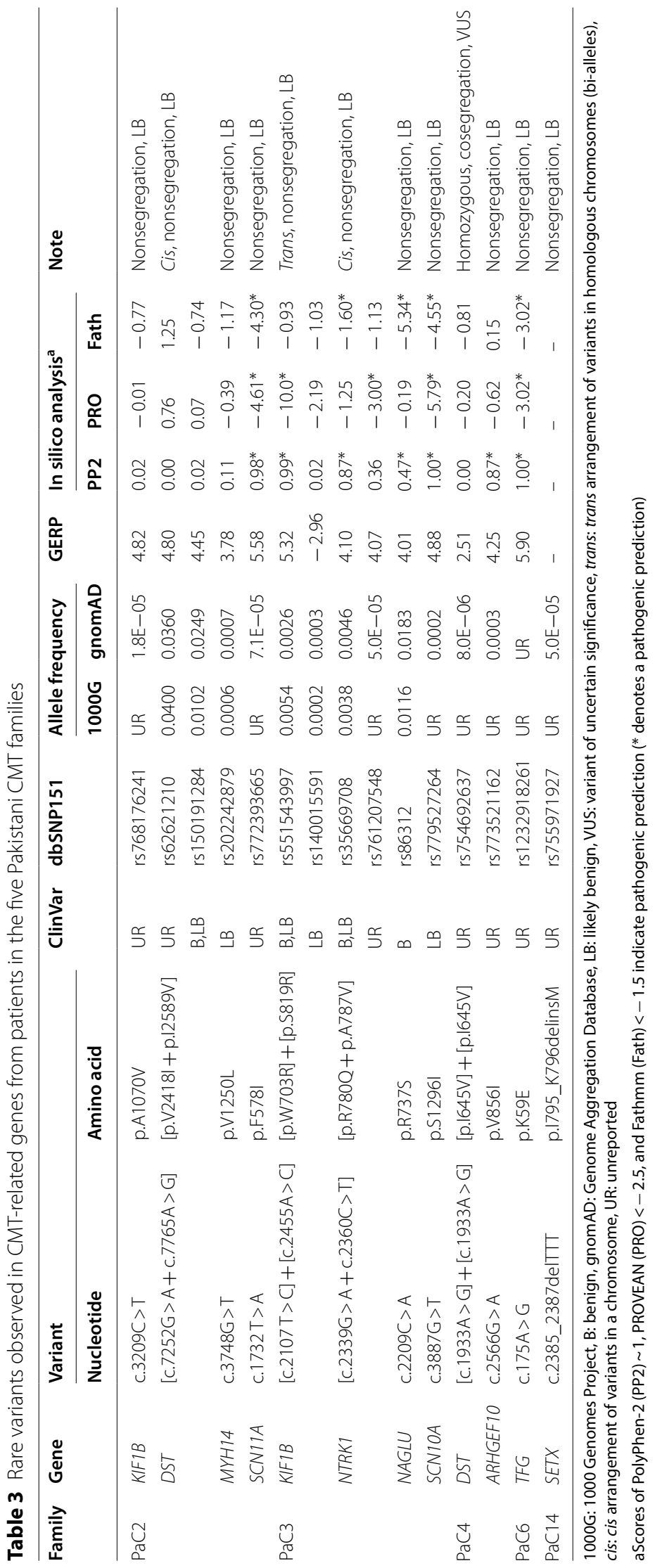



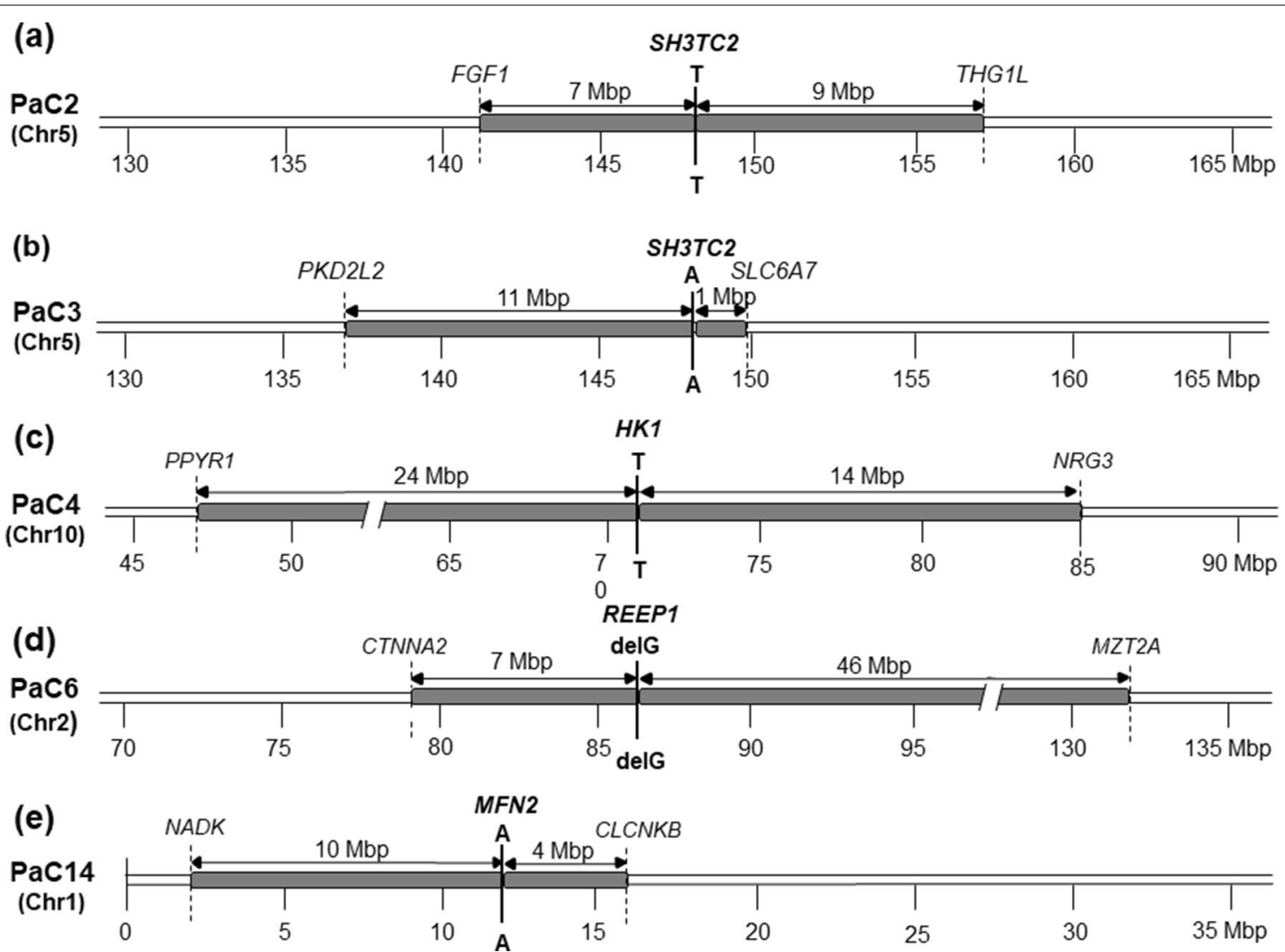

Fig. 3 Homozygosity mapping of the chromosomal regions around the pathogenic mutations for the affected individuals from the consanguineous Pakistani CMT families. Genes and SNP numbers located at the ends of the homozygous blocks (HBs) and their approximate chromosomal positions (indicating by Mbp) are shown at the top and bottom of the maps, respectively. a $16 \mathrm{Mbp}$ HB from FGF1 to THG1L in the PaC2 family with SH3TC2 mutation. b $12 \mathrm{Mbp} \mathrm{HB}$ from PKD2L2 to SLC6A7 in the PaC3 family with SH3TC2 mutation. c 38 Mbp HB from PPYR1 to NRG3 in the PaC4 family with HK1 mutation. d $53 \mathrm{Mbp} \mathrm{HB}$ from CTNNA2 to MZT2A in the PaC6 family with REEP1 mutation. e 14 Mbp HB from NADK to CLCNKB in the PaC14 family with MFN2 mutation

(ER)-mitochondrial interactions [34]. Reep1 null mice displayed alterations in ER morphogenesis and lipid abnormalities [35]. It is known that the REEP1 mutations exhibited considerable phenotypic heterogeneity [36]. Dominant REEP1 mutations have been reported to cause dHMN5B (DSMA5B) and SPG21 with the onset ages falling in either the first or second decades [24, 25]. Recently, a recessive REEP1 mutation (p.Phe62Lysfs"23) was reported in a 5-year-old Lebanese boy manifesting a similar SMARD phenotype [26]. The affected boy presented foot deformity and contractures of the distal phalanges at the time of birth. This was similar to PaC6 family who harbored a p.Gly83Alafs" 44 mutation resulting in a premature termination and exhibited a similar onset age, although some clinical features were different. In the nerve conduction studies, none of the motor nerves were evoked, but all sensory nerves showed normal SNAPs and SNCVs. From the clinical and NCV findings, this patient's symptoms are apparently similar to those of SMARD.

MFN2 encodes mitofusin 2 which plays an important role in maintaining equilibrium between mitochondrial fusion and fission [37]. Most MFN2 mutations have been reported to cause dominant CMT2A2A [27]. However, some homozygous or compound heterozygous mutations cause recessive CMT2A2B (MIM 617087) that is associated with more severe and earlier onset phenotypes. Nicholson et al. suggested that CMT2A2B may be semidominant, and carriers harboring a single mutant allele may manifest a weak phenotype with incomplete penetrance [29]. Our patient harboring homozygous MFN2 mutation manifested relatively early onset (5 years old) and severe phenotypes, corresponding to CMT2A2B phenotypes. Additionally, vocal cord paralysis seen in the affected boy has been occasionally reported in CMT2A patients harboring MFN2 mutations [38, 39]. The patient showed no symptom of optic atrophy which 
is a characteristic of CMT6A caused by MFN2 mutations until the time of the first examination (7 years old). His parents harboring the heterozygous MFN2 mutation were apparently unaffected; however, clinical and electrophysiological tests were not performed. Therefore, it is likely that they might be mildly affected because of the semidominant nature of the mutation.

Next generation sequencing data can be used for homozygosity mapping of recessive traits in consanguineous pedigrees [40]. When we performed homozygosity mapping around the pathogenic mutations using SNPs from the WES data of the affected members, all the examined families showed same haplotype blocks between two homologous chromosomes. This suggests that both alleles of the homozygous mutations identified in each family originated from a single ancestor. Although a few CMT cases were investigated in this study, the frequency of recessive patients with homozygous mutations was certainly higher than that in other countries, such as United Kingdom, United States, Italy, and Japan [5, 41, 42]. Mutations in MFN2 are well recognized as the cause of dominant CMT2; however, recessive homozygous mutations have been rarely reported.

\section{Conclusions}

In conclusion, we identified five pathogenic or likely pathogenic mutations in consanguineous Pakistani families with early onset CMT. All the mutations were novel, and the genotype-phenotype correlations were confirmed. We believe that our findings will expand our understanding of the genetic basis of peripheral neuropathy and improve molecular diagnostics and treatment options.

\begin{abstract}
Abbreviations
ACMG: American College of Medical Genetics and Genomics; CMAP: Compound muscle action potential; CMT: Charcot-Marie-Tooth disease; dHMN: Distal hereditary motor neuropathy; dSMA5B: Distal spinal muscular atrophy type 5B; ER: Endoplasmic reticulum; FDS: Functional disability scale; GERP: Genomic evolutionary rate profiling score; HB: Homozygous block; HMSN: Hereditary motor and sensory neuropathy; HSAN: Hereditary sensory and autonomic neuropathy; MAF: Minor allele frequency; MNCV: Motor nerve conduction velocity; NCV: Nerve conduction velocity; OMM: Outer membrane of mitochondria; SMARD: Spinal muscular atrophy with respiratory distress; SNAP: Sensory nerve action potential; SNCV: Sensory nerve conduction velocity; TPR: Tetratricopeptide repeat; WES: Whole exome sequencing.
\end{abstract}

\section{Acknowledgements}

We would like to thank the patients and their families for their consent of participation and sample donation.

\footnotetext{
Authors' contributions

Conceptualization: KWC, SK; Sampling: SK, RN, AK, SP; Genetic and molecular investigation: YJC, SOL, HJC, JHP, SP; Clinical data analysis: RN, AK, BOC; Funding acquisition: $\mathrm{KWC}, \mathrm{BOC}$; Writing original draft preparation: $\mathrm{BOC}$ KWC; Review and editing: all authors. All authors read and approved the final manuscript.
}

\section{Funding}

This work was supported by grants from the National Research Foundation (2019R1A2C1087547, 2020M3H4A1A03084600, and 2021R1A4A2001389) and the Korean Health Technology R\&D Project, Ministry of Health and Welfare (HI14C3484 and HI20C0039), Republic of Korea. The funding bodies provide us with financial support for data analysis and interpretation, language editing service and administrative work.

\section{Availability of data and materials}

The datasets generated and analyzed during the current study are available in the NCBI Sequence Read Archive Database (Accession No.: PRJNA735898; https://www.ncbi.nlm.nih.gov/Traces/study/?acc=PRJNA735898). The web links of the relevant datasets were as follows: hg19 (http://genome.ucsc.edu/), 1000 Genomes project (http://www.1000genomes.org/), dbSNP (http://www. ncbi.nlm.nih.gov/snp), gnomAD (https://gnomad.broadinstitute.org/about), ClinVar (https://www.ncbi.nlm.nih.gov/clinvar/), and OMIM (http://omim.org).

\section{Declarations}

Ethics approval and consent to participate

This study was approved by the Institutional Review Boards for Kongju National University (KNU_RB_2018-06), Sungkyunkwan University, Samsung Medical Center (2014-08-057-002), COMSATS University Islamabad, Sahiwal (CUI-SWL-09-062-2018), and The Children Hospital and Institute of Child Health Multan (CHMP-2018-023-0014). All participants were recruited from The Children Hospital and Institute of Child Health Multan, Pakistan, and provided written informed consent. For the minors involved in the study, the written consent was provided by their parents.

\section{Consent for publication}

Written consent was provided from all the participants for publication of their clinical data. The rewritten content was also obtained for minors from the parents.

\section{Competing interests}

The authors declare that they have no competing interests.

\section{Author details}

${ }^{1}$ Department of Biosciences, COMSATS University Islamabad, Sahiwal, Pakistan. ${ }^{2}$ Department of Biological Sciences, Kongju National University, 56 Gongjudaehakro, Gongju 32588, Korea. ${ }^{3}$ Department of Pediatric Neurology, The Children Hospital and Institute of Child Health, Multan, Pakistan. ${ }^{4}$ Department of Zoology, The Women University, Multan, Pakistan. ${ }^{5}$ Department of Neurology, Samsung Medical Center, Sungkyunkwan University School of Medicine, 81 Irwon-ro, Gangnam-gu, Seoul 06351, Korea.

Received: 15 February 2021 Accepted: 18 June 2021

Published online: 30 June 2021

\section{References}

1. Barreto LC, Oliveira FS, Nunes PS, de França Costa IM, Garcez CA, Goes GM, et al. Epidemiologic study of Charcot-Marie-Tooth disease: a systematic review. Neuroepidemiology. 2016;46:157-65.

2. Saporta MA, Shy ME. Inherited peripheral neuropathies. Neurol Clin. 2013;31:597-619.

3. Harding AE, Thomas PK. Genetic aspects of hereditary motor and sensory neuropathy (types I and II). J Med Genet. 1980;17:329-36.

4. Fridman V, Bundy B, Reilly MM, Pareyson D, Bacon C, Burns J, et al. CMT subtypes and disease burden in patients enrolled in the inherited neuropathies consortium natural history study: a cross-sectional analysis. J Neurol Neurosurg Psychiatry. 2015;86:873-8.

5. Gentile L, Russo M, Fabrizi GM, Taioli F, Ferrarini M, Testi S, et al. CharcotMarie-Tooth disease: experience from a large Italian tertiary neuromuscular center. Neurol Sci. 2020;241:1239-43.

6. Houlden H, Laura M, Wavrant-De Vrièze F, Blake J, Wood N, Reilly MM. Mutations in the HSP27 (HSPB1) gene cause dominant, recessive, and sporadic distal HMN/CMT type 2. Neurology. 2008;71:1660-8. 
7. Pedurupillay CR, Amundsen SS, Barøy T, Rasmussen M, Blomhoff A, Stadheim BF, et al. Clinical and molecular characteristics in three families with biallelic mutations in IGHMBP2. Neuromuscul Disord. 2016;26:570-5.

8. Zambon AA, Natali Sora MG, Cantarella G, Cerri F, Quattrini A, Comi G, et al. Vocal cord paralysis in Charcot-Marie-Tooth type 4b1 disease associated with a novel mutation in the myotubularin-related protein 2 gene: a case report and review of the literature. Neuromuscul Disord. 2017;27:487-91.

9. Wright GC, Brown R, Grayton H, Livingston JH, Park SM, Parker APJ, et al. Clinical and radiological characterization of novel FIG4-related combined system disease with neuropathy. Clin Genet. 2020;98:147-54.

10. Birouk N, LeGuern E, Maisonobe T, Rouger H, Gouider R, Tardieu S, et al. X-linked Charcot-Marie-Tooth disease with connexin 32 mutations: clinical and electrophysiologic study. Neurology. 1998;50:1074-82.

11. Richards S, Aziz N, Bale S, Bick D, Das S, Gastier-Foster J, et al. Standards and guidelines for the interpretation of sequence variants: a joint consensus recommendation of the American College of Medical Genetics and Genomics and the Association for Molecular Pathology. Genet Med. 2015;17:405-24

12. Cheng J, Randall A, Baldi P. Prediction of protein stability changes for single-site mutations using support vector machines. Proteins. 2006;62:1125-32

13. Adzhubei IA, Schmidt S, Peshkin L, Ramensky VE, Gerasimova A, Bork P, et al. A method and server for predicting damaging missense mutations. Nat Methods. 2010;7:248-9.

14. Choi Y, Chan AP. PROVEAN web server: a tool to predict the functional effect of amino acid substitutions and indels. Bioinformatics. 2015;31:2745-7.

15. Shihab HA, Gough J, Cooper DN, Stenson PD, Barker GLA, Edwards KJ, et al. Predicting the functional, molecular and phenotypic consequences of amino acid substitutions using hidden Markov models. Hum Mutat. 2013;34:57-65.

16. Davydov EV, Goode DL, Sirota M, Cooper GM, Sidow A, Batzoglou S. Identifying a high fraction of the human genome to be under selective constraint using GERP++. PLoS Comput Biol. 2010;6:1001025.

17. Park HR, Kanwal S, Lim SO, Nam DE, Choi YJ, Chung KW. Homozygous mutations in Pakistani consanguineous families with prelingual nonsyndromic hearing loss. Mol Biol Rep. 2020;47:9979-85.

18. Senderek J, Bergmann C, Stendel C, Kirfel J, Verpoorten N, De Jonghe $\mathrm{P}$, et al. Mutations in a gene encoding a novel SH3/TPR domain protein cause autosomal recessive Charcot-Marie-Tooth type 4C neuropathy. Am J Hum Genet. 2003;73:1106-19.

19. Lupski JR, Reid JG, Gonzaga-Jauregui C, Rio Deiros D, Chen DCY, Nazareth $L$, et al. Whole-genome sequencing in a patient with Charcot-MarieTooth neuropathy. New Engl J Med. 2010;362:1181-91.

20. Azzedine H, Ravisé N, Verny C, Gabrëels-Festen A, Lammens M, Grid D, et al. Spine deformities in Charcot-Marie-Tooth 4C caused by SH3TC2 gene mutations. Neurology. 2006;67:602-6.

21. Yger M, Stojkovic T, Tardieu S, Maisonobe T, Brice A, Echaniz-Laguna A, et al. Characteristics of clinical and electrophysiological pattern of Charcot-Marie-Tooth 4C. J Peripher Nerv Syst. 2012;17:112-22.

22. Hantke J, Chandler D, King R, Wanders RJA, Angelicheva D, Tournev I, et al. A mutation in an alternative untranslated exon of hexokinase 1 associated with hereditary motor and sensory neuropathy-Russe (HMSNR). Eur J Hum Genet. 2009;17:1606-14.

23. Sevilla T, Martinez-Rubio D, Marquez C, Paradas C, Colomer J, Jaijo T, et al. Genetics of the Charcot-Marie-Tooth disease in the Spanish Gypsy population: the hereditary motor and sensory neuropathy-Russe in depth. Clin Genet. 2013;83:565-70

24. Beetz C, Pieber TR, Hertel N, Schabhuttl M, Fischer C, Trajanoski S, et al. Exome sequencing identifies a REEP1 mutation involved in distal hereditary motor neuropathy type V. Am J Hum Genet. 2012;91:139-45.
25. Züchner S, Wang G, Tran-Viet K-N, Nance MA, Gaskell PC, Vance JM, et al. Mutations in the novel mitochondrial protein REEP1 cause hereditary spastic paraplegia type 31. Am J Hum Genet. 2006;79:365-9.

26. Schottmann G, Seelow D, Seifert F, Morales-Gonzalez S, Gill E, von Au $\mathrm{K}$, et al. Recessive REEP1 mutation is associated with congenital axonal neuropathy and diaphragmatic palsy. Neurol Genet. 2015;1:32.

27. Züchner S, Mersiyanova IV, Muglia M, Bissar-Tadmouri N, Rochelle J, Dadali EL, et al. Mutations in the mitochondrial GTPase mitofusin 2 cause Charcot-Marie-Tooth neuropathy type 2A. Nat Genet. 2004;36:449-51.

28. Züchner S, De Jonghe P, Jordanova A, Claeys KG, Guergueltcheva V, Cherninkova $S$, et al. Axonal neuropathy with optic atrophy is caused by mutations in mitofusin 2. Ann Neurol. 2006;59:276-81.

29. Nicholson GA, Magdelaine C, Zhu D, Grew S, Ryan MM, Sturtz F, et al. Severe early-onset axonal neuropathy with homozygous and compound heterozygous MFN2 mutations. Neurology. 2008;70:1678-81.

30. Edvardson S, Cinnamon Y, Jalas C, Shaag A, Maayan C, Axelrod FB, Elpeleg O. Hereditary sensory autonomic neuropathy caused by a mutation in dystonin. Ann Neurol. 2012;71:569-72.

31. Arnaud E, Zenker J, de Preux Charles A-S, Stendel C, Roos A, Medard J-J, et al. SH3TC2/KIAA1985 protein is required for proper myelination and the integrity of the node of Ranvier in the peripheral nervous system. Proc Natl Acad Sci USA. 2009;106:17528-33.

32. Colomer J, Gooding R, Angelicheva D, King RHM, Guillen-Navarro E, Parman Y, et al. Clinical spectrum of CMT4C disease in patients homozygous for the p.Arg1109X mutation in SH3TC2. Neuromuscul Disord. 2006;16:449-53.

33. Sullivan LS, Koboldt DC, Bowne SJ, Lang S, Blanton SH, Cadena E, et al. A dominant mutation in hexokinase 1 (HK1) causes retinitis pigmentosa. Invest Ophthal Vis Sci. 2014;55:7147-58.

34. Lim Y, Cho I-T, Schoel LJ, Cho G, Golden JA. Hereditary spastic paraplegialinked REEP1 modulates endoplasmic reticulum/mitochondria contacts. Ann Neurol. 2015;78:679-96.

35. Renvoisé B, Malone B, Falgairolle M, Munasinghe J, Stadler J, Sibilla C, et al. Reep1 null mice reveal a converging role for hereditary spastic paraplegia proteins in lipid droplet regulation. Hum Mol Genet. 2016;25:5111-25.

36. Maroofian R, Behnam M, Kaiyrzhanov R, Salpietro V, Salehi M, Houlden H. Further supporting evidence for REEP1 phenotypic and allelic heterogeneity. Neurol Genet. 2019;5:379.

37. Santel A, Fuller MT. Control of mitochondrial morphology by a human mitofusin. J Cell Sci. 2001;114:867-74.

38. Bombelli F, Stojkovic T, Dubourg O, Echaniz-Laguna A, Tardieu S, Larcher $\mathrm{K}$, et al. Charcot-Marie-Tooth disease type 2A: from typical to rare phenotypic and genotypic features. JAMA Neurol. 2014;71:1036-42.

39. Ando M, Hashiguchi A, Okamoto $Y$, Yoshimura A, Hiramatsu Y, Yuan J, et al. Clinical and genetic diversities of Charcot-Marie-Tooth disease with MFN2 mutations in a large case study. J Peripher Nerv Syst. 2017:22:191-9.

40. Wakeling MN, Laver TW, Wright CF, De Franco E, Stals KL, Patch AM, et al. Homozygosity mapping provides supporting evidence of pathogenicity in recessive Mendelian disease. Genet Med. 2019;21:982-6.

41. Cortese A, Wilcox JE, Polke JM, Poh R, Skorupinska M, Rossor AM, et al. Targeted next-generation sequencing panels in the diagnosis of Charcot-Marie-Tooth disease. Neurology. 2020;94:e51-61.

42. Yoshimura A, Yuan JH, Hashiguchi A, Ando M, Higuchi Y, Nakamura T, et al Genetic profile and onset features of 1005 patients with Charcot-MarieTooth disease in Japan. J Neurol Neurosurg Psychiatry. 2019;90:195-202.

\section{Publisher's Note}

Springer Nature remains neutral with regard to jurisdictional claims in published maps and institutional affiliations. 